\title{
A Study of the Coulomb Dissociation of ${ }^{8} B$ and the ${ }^{7} B e(p, \gamma)^{8} B$ Reaction
}

\author{
Carlos A. Bertulani ${ }^{a}$, and Moshe Gai ${ }^{b}$ \\ a Instituto de Física, Universidade Federal do Rio de Janeiro \\ 21945-970 Rio de Janeiro, RJ, Brazil. E-mail: bertu@if.ufrj.br \\ ${ }^{b}$ Department of Physics, U46, University of Connecticut \\ 2152 Hillside Rd., Storrs, CT 06269-3046, USA. E-mail: gai@uconnvm.uconn.edu
}

(August 7, 2018)

\begin{abstract}
We study the breakup reactions of ${ }^{8} B$ projectiles in high energy (50 and $250 \mathrm{MeV} / \mathrm{u}$ ) collisions with heavy nuclear targets $\left({ }^{208} \mathrm{~Pb}\right)$. The intrinsic nuclear wave functions are calculated using a simple model, as well as a simple optical potential. We demonstrate that nuclear effects are negligible and evaluate the contributions of various (E1, E2 and M1) multipolarities. A good agreement with measured data is obtained with insignificant M1 contribution (at $50 \mathrm{MeV} / \mathrm{u}$ ) and very small E2 contribution.
\end{abstract}

PACS numbers: 25.60.P, 25.40, 25.20, 26.65

\section{INTRODUCTION}

The use of the Coulomb dissociation method [1:22 has proven to be a useful tool for extracting radiative capture reaction cross section of relevance for nuclear astrophysics. In particular it appears that the Coulomb dissociation of ${ }^{8} B$ is very useful [3] for elucidating the most uncertain nuclear input to the standard solar model - the formation of ${ }^{8} B$ via the ${ }^{7} B e(p, \gamma)^{8} B$ reaction. However, a few lingering questions still need to be addressed, including the importance of nuclear excitations for the kinematics of the RIKEN [4] and GSI [5] experiments, performed at approximately 50 and $250 \mathrm{MeV} / \mathrm{u}$, respectively, as well as the relative importance of the various E1, E2 and M1 electromagnetic excitations. In this paper we attempt to resolve these issues by using a relatively simple but still realistic nuclear model that however yields a very good agreement with data and suggest that nuclear excitations as well as E2 and M1 excitations are negligible for the kinematical conditions of the RIKEN and GSI experiments.

\section{TRANSITION DENSITIES}

We use for ${ }^{8} \mathrm{~B}$ a similar model as in ref. [6], assuming that the $J_{0}=2^{+}$ground state can be described as a $j_{0}=p_{3 / 2}$ proton coupled to the $I_{c}=3 / 2^{-}$ground state of the ${ }^{7} \mathrm{Be}$ core. The spectroscopic factor for this configuration was taken as unity. The single particle states, $\Psi_{J M}$, are found by solving the Schrödinger equation with spin-orbit term and matching to asymptotic Coulomb waves. The parameters of the potentials are given in table 1 of ref. [6].

A multipole expansion of the transition density, $\delta \rho$, yields

$$
\delta \rho(\mathbf{r}) \equiv \Psi_{J M}^{*}(\mathbf{r}) \Psi_{J_{0} M_{0}}(\mathbf{r})=\sum_{\lambda \mu} \delta \rho_{\lambda \mu}^{(J M)}(r) Y_{\lambda \mu}(\hat{\mathbf{r}}),
$$

where

$$
\delta \rho_{\lambda \mu}^{(J M)}(r)=\int \Psi_{J M}^{*}(\mathbf{r}) Y_{\lambda \mu}^{*}(\hat{\mathbf{r}}) \Psi_{J_{0} M_{0}}(\mathbf{r}) d \Omega,
$$

and $\mid J_{0} M_{0}>(\mid J M>)$ denote the initial (final) state of the projectile.

The electromagnetic transition matrix for the multipolarity $\pi \lambda \mu$ is given by 


$$
\begin{aligned}
\left\langle J M\left|\mathcal{M}_{\pi \lambda,-\mu}\right| J_{0} M_{0}\right\rangle & =\int d r r^{\lambda} \delta \rho_{E \lambda \mu}^{(J M)} \quad \text { (electric); } \\
& =\int d r r^{\lambda-1} \delta \rho_{M \lambda \mu}^{(J M)} \quad \text { (magnetic) }
\end{aligned}
$$

where, for electric multipole transitions

$$
\begin{aligned}
\delta \rho_{E \lambda \mu}^{(J M)}(r) & =\frac{1}{2 \sqrt{4 \pi}}(-1)^{I_{c}+J_{0}+\lambda+j_{0}+l+l_{0}+\mu} e_{E} \frac{\hat{J_{0}} \hat{\lambda} \hat{j_{0}}}{\hat{j}} \\
& \times\left\langle J_{0} M_{0} \lambda \mu \mid J M\right\rangle\left\langle j_{0} \frac{1}{2} \lambda 0 \mid j \frac{1}{2}\right\rangle\left\{\begin{array}{ccc}
j & J & I_{c} \\
J_{0} & j_{0} & \lambda
\end{array}\right\} \delta \rho_{E_{x} l j l_{0} j_{0}}^{(J)},
\end{aligned}
$$

where $\hat{l}=\sqrt{2 l+1}, e_{E}=e\left[(1 / 2)^{\lambda}+(-1 / 8)^{\lambda}\right]$, and

$$
\delta \rho_{E_{x} l j ; l_{0} j_{0}}^{(J)}(r)=r^{2} R_{E_{x} l j}^{(J) *}(r) R_{l_{0} j_{0}}^{\left(J_{0}\right)}(r) .
$$

Above, $R_{l_{0} j_{0}}^{\left(J_{0}\right)}(r)$ is the radial wave function for the ground-state and $R_{E_{x} l j}^{(J)}(r)$ is the radial wave function for a state in the continuum with excitation energy $E_{x}$.

For magnetic dipole transitions

$$
\begin{aligned}
\delta_{M \lambda \mu}^{(J M)}(r) & =\mu_{N}(-1)^{j_{0}+I_{c}+J_{0}+1} \frac{\hat{J_{0}}}{\hat{l_{0}}}\left\langle J_{0} M_{0} \lambda \mu \mid J M\right\rangle\left\{\begin{array}{ccc}
j & J & I_{c} \\
J_{0} & j_{0} & 1
\end{array}\right\} \\
& \times\left\{e_{M}\left[2 \frac{\tilde{j_{0}}}{\hat{l_{0}}}\left(l_{0} \delta_{j_{0}, l+\frac{1}{2}}+(l+1) \delta_{j_{0}, l-\frac{1}{2}}\right)+(-1)^{l_{0}+\frac{1}{2}-j} \frac{\hat{j_{0}}}{\hat{2}} \delta_{j_{0}, l_{0} \pm \frac{1}{2}} \delta_{j, l \mp \frac{1}{2}}\right]\right. \\
& \left.+g_{p}\left[(-1)^{l_{0}+\frac{1}{2}-j_{0}} \frac{\tilde{j_{0}}}{\hat{l_{0}}} \delta_{j_{0}, j}-(-1)^{l_{0}+\frac{1}{2}-j} \frac{\hat{j_{0}}}{\hat{2} \hat{l_{0}}} \delta_{j_{0}, l \pm \frac{1}{2}} \delta_{j, l \mp \frac{1}{2}}\right]\right\} \delta \rho_{E_{x} l j ; l_{0} j_{0}}^{(J)}(r) \\
& +\mu_{c}(-1)^{I_{c}+j_{0}+J+1}\left\langle J_{0} M_{0} \lambda \mu \mid J M\right\rangle \hat{J}_{0} \hat{J} \hat{I}_{c} \tilde{I}_{c}\left\{\begin{array}{ccc}
I_{c} & J & j_{0} \\
J_{0} & I_{c} & 1
\end{array}\right\} \delta \rho_{E_{x} l j ; l_{0} j_{0}}^{(J)}(r)
\end{aligned}
$$

where $e_{M}=3 / 2, \mu_{c}=-1.7 \mu_{N}, \mu_{N}=e \hbar / 2 m_{p} c, m_{p}$ is the proton mass, and $\tilde{l}=\sqrt{l(l+1)}$.

In addition to the electric and magnetic transitions due to Coulomb excitation of the projectile, we will also consider the transitions induced by the nuclear field of the target in peripheral collisions. The nuclear induced transition density is built also as in eqs. $(1,2)$, and for isoscalar excitations (we will not consider nuclear isovector excitations for reasons explained below) we can write the transition density as $\delta \rho_{N \lambda \mu}^{(J M)}=\delta \rho_{E \lambda \mu}^{(J M)} / e_{E}$.

The transition densities, $\delta \rho_{E_{x} l j j l_{0} j_{0}}^{(J)}(r)$, are shown in figures $1(\mathrm{a})$ and $1(\mathrm{~b})$. In figure 1(a) we show the transition densities to the $J=1^{+}$, and $J=3^{+}$, resonances at $E_{x}=0.63 \mathrm{MeV}$, and $E_{x}=2.17 \mathrm{MeV}$, respectively. For convenience, the transition densities have been integrated over the width of the resonance. Also shown in this figure (dotted line) is the transition density obtained by the Tassie model (see, e.g., ref. [7]): $\delta \rho_{\text {Tassie }}(r)=(\beta r) d \rho / d r$, where $\rho(r)$ is the ground-state density of the ${ }^{8} B$, taken as $\rho(r)=\rho_{0}\left[1+\alpha(r / a)^{2}\right] \exp \left\{-r^{2} / a^{2}\right\}$, with $\alpha=0.631 \mathrm{fm}$ and $a=1.77 \mathrm{fm}$. The deformation parameter, $\beta$, is chosen so that the Tassie transition density is normalized to the peak of the transition density to the $J=3^{+}$state. We see that the transition densities extend further out than the Tassie transition density. This is even more visible for the transition densities to the non-resonant p- and f-waves, as shown in figure 1(b), for $E_{x}=350 \mathrm{keV}$. These transition densities extend to very large radial values. This is an important result since it invalidates calculations based on the Tassie model for the transition densities in the case of halo nuclei, as was pointed out in ref. [8]. 


\section{OPTICAL AND TRANSITION POTENTIALS}

Since there is no data for the elastic scattering of ${ }^{8} B$ on $P b$ targets at the energies that we want to consider, we construct an optical potential using an effective interaction of the M3Y type [9,10] modified so as to reproduce the energy dependence of total reaction cross sections, i.e. [10],

$$
t(E, s)=-i \frac{\hbar v}{2 t_{0}} \sigma_{N N}(E)[1-i \alpha(E)] t(s)
$$

where $t_{0}=421 \mathrm{MeV}$ is the volume integral of the M3Y interaction $t(s), v$ is the projectile velocity, $\sigma_{N N}$ is the nucleonnucleon cross section, and $\alpha$ is the real-to-imaginary ratio of the forward nucleon-nucleon scattering amplitude. The optical potential is given by

$$
U(E, \mathbf{R})=\int d^{3} r_{1} d^{3} r_{2} \rho_{P}\left(\mathbf{r}_{1}\right) \rho_{T}\left(\mathbf{r}_{2}\right) t(E, s)
$$

where $\mathbf{s}=\mathbf{R}+\mathbf{r}_{2}-\mathbf{r}_{1}$, and $\rho_{T}\left(\rho_{P}\right)$ is the ground state density of the target (projectile).

According to this model, the optical potential is about two times smaller at $250 \mathrm{MeV} /$ nucleon compared to 50 $\mathrm{MeV} /$ nucleon. The optical potentials generated in this way will be used to obtain the distorted waves in the eikonal approximation (see next section).

The transition potential for excitations of the projectile is given by

$$
\Delta U(E, \mathbf{R})=\int d^{3} r_{1} d^{3} r_{2} \delta \rho_{P}\left(\mathbf{r}_{1}\right) \rho_{T}\left(\mathbf{r}_{2}\right) t(E, s)
$$

A multipole expansion of the transition potential yields

$$
\Delta U(E, \mathbf{R})=\sum_{\lambda \mu} \delta U_{\lambda \mu}^{(J M)}(E, R) Y_{\lambda \mu}(\hat{\mathbf{R}})
$$

where

$$
\delta U_{\lambda \mu}^{(J M)}(E, R)=\int d r r^{2} I_{\lambda}(E, R, r) \delta \rho_{N \lambda \mu}^{(J M)}(r)
$$

with

$$
I_{\lambda}\left(E, R, r_{2}\right)=(2 \pi)^{2} \int d\left(\cos \theta_{1}\right) d\left(\cos \theta_{2}\right) d r_{1} r_{1}^{2} t(E, s) P_{\lambda}\left(\cos \theta_{2}\right),
$$

where $\theta_{1}$ is the angle between $\mathbf{r}_{1}$ and $\mathbf{R}+\mathbf{r}_{2}$, and $\theta_{2}$ is the angle between $\mathbf{r}_{2}$ and $\mathbf{R}$.

In figures $2(\mathrm{a})$ and $2(\mathrm{~b})$ we show the transition potentials

$$
\delta U_{E_{x} l j ; l_{0} j_{0}}(R)=\int d r r^{2} I_{2}(R, r) \delta \rho_{E_{x} l j ; l_{0} j_{0}}(r)
$$

as a function of the radial distance, for ${ }^{8} B$ projectiles incident on $P b$ at $\mathrm{E}=50 \mathrm{MeV} /$ nucleon. In figure $2(\mathrm{a})$ we show the real part of the transition potentials, integrated over the width of the $J=1^{+}$(solid line) and $J=3^{+}$(dashed line) resonances. They are compared with the Copenhagen model (dashed line) for the transition potential (called by "standard potential" in ref. [7]), i.e., $\delta U_{C o p}=(\beta r) d U_{o p t} / d r$, with an arbitrary value for the deformation parameter $\beta$. Again we see that the transition potentials have a quite different radial dependence than the Copenhagen transition potential model. For the non-resonant $\mathrm{p}$ and $\mathrm{f}$ waves this difference is even more pronounced, as we see in figure 2(b), for $E_{x}=350 \mathrm{keV}$. According to eqs. (3.1,3.3), at $250 \mathrm{MeV} /$ nucleon the transition potentials have the same shape as those presented in figures $2(\mathrm{a})$ and $2(\mathrm{~b})$, but are about twice smaller in magnitude since the effective interaction $t(E, s)$ is reduced by nearly the same amount. 


\section{NUCLEAR AND COULOMB EXCITATION CROSS SECTIONS}

The amplitude for the nuclear excitation of high energy projectiles is given by

$$
\begin{aligned}
f_{N} & =-\frac{\mu_{P T}}{2 \pi \hbar^{2}} \int d^{3} R \Psi^{(-) *}(\mathbf{R}) \Psi^{(+)}(\mathbf{R}) \Delta U(\mathbf{R}) \\
& =\sum_{\lambda \mu} f_{N \lambda \mu}^{(J M)},
\end{aligned}
$$

where $\mu_{P T}$ is the reduced mass of the projectile+target system, $\Psi^{(-)}\left(\Psi^{(+)}\right)$is the incoming (outgoing) scattering wave of the system, and

$$
f_{N \lambda \mu}^{(J M)}=-\frac{\mu_{P T}}{2 \pi \hbar^{2}} \int d^{3} R \Psi^{(-) *}(\mathbf{R}) \Psi^{(+)}(\mathbf{R}) \delta U_{\lambda \mu}^{(J M)}(\mathbf{R}) Y_{\lambda \mu}(\hat{\mathbf{R}}) .
$$

For high energy projectiles, we can use the eikonal approximation $(Q=2 k \sin (\theta / 2))$,

$$
\Psi^{(-) *} \Psi^{(+)}=\exp \{i \mathbf{Q} \cdot \mathbf{R}+i \chi(b)\}
$$

with the eikonal phase given by

$$
\chi(b)=2 \eta \ln (k b)-\frac{1}{\hbar v} \int_{-\infty}^{\infty} d z U_{o p t}(R)
$$

where $\eta=Z_{P} Z_{T} e^{2} / \hbar v, k$ is the projectile momentum, and $R=\sqrt{b^{2}+z^{2}}$. The optical potential, $U_{o p t}$, in the above equation is given by eq. (3.2).

Following ref. [11], the Coulomb amplitude is given by

$$
f_{C}=\sum_{\lambda \mu} f_{C \lambda \mu}
$$

where

$$
\begin{aligned}
& f_{C \lambda \mu}=i^{1+\mu} \frac{Z_{T} e \mu_{P T}}{\hbar^{2}}\left(\frac{E_{x}}{\hbar c}\right)^{\lambda} \sqrt{\lambda+1} \exp \{-i \mu \phi\} \Omega_{\mu}(q) \\
& \times G_{\pi \lambda \mu}\left(\frac{c}{v}\right)\left\langle J M\left|\mathcal{M}_{\pi \lambda,-\mu}\right| J_{0} M_{0}\right\rangle \\
& \Omega_{\mu}(q)=\int_{0}^{\infty} d b b J_{\mu}(q b) K_{\mu}\left(\frac{E_{x} b}{\gamma \hbar v}\right) \exp i \chi(b)
\end{aligned}
$$

$J_{\mu}\left(K_{\mu}\right)$ is the cylindrical (modified) Bessel function of order $\mu$, and the functions $G_{\pi \lambda \mu}(c / v)$ are tabulated in ref. [12].

The cross sections for Coulomb plus nuclear excitation are given by

$$
\frac{d \sigma_{\lambda}^{J}}{d \Omega d E_{x}}=\frac{1}{2 J_{0}+1} \sum_{M_{0}, M}\left|f_{C}^{\lambda \mu}+f_{N}^{\lambda \mu}\right|^{2}
$$

Further simplifications can be obtained by noticing that, since the M3Y interaction does not depend on isospin, the nuclear isovector excitations are absent from $f_{N}$. This is well justified, since even in the case when isovector excitations can be reached, they are of minor importance compared to the isoscalar ones [13. Thus, the nuclear excitation of isovector dipole modes $(\lambda=1)$ will be suppressed and we can neglect this multipolarity (also because intrinsic isoscalar dipole excitations do not exist) in the sum of eq. (4.8). Moreover, angular momentum selection rules imply that the $J=1^{+}$and $J=3^{+}$do not contribute to $\lambda=0$ excitation amplitudes. Also, non-resonant $p_{1 / 2}$ 
waves cannot be reached via $\lambda=0$ excitation. Thus, nuclear monopole $(\lambda=0)$ excitations will also be absent from this sum.

For the Coulomb amplitude we will consider E1, E2, and $M 1$ excitations (monopole, E0, excitations cannot be achieved in Coulomb excitation). Thus the cross section including both Coulomb and nuclear excitation becomes

$$
\frac{d \sigma_{\pi \lambda}^{J}}{d \Omega d E_{x}}=\frac{1}{2 J_{0}+1} \sum_{M_{0}, M}\left[\left|f_{C}^{(E 1)}\right|^{2}+\left|f_{C}^{(M 1)}\right|^{2}+\left|f_{N}^{(2)}+f_{C}^{(E 2)}\right|^{2}\right]
$$

The nuclear-Coulomb interference will only appear for quadrupole excitations.

\section{RESULTS}

In figure 3(a) we plot the cross section for the nuclear excitation of ${ }^{8} \mathrm{~B}$ projectiles incident on $\mathrm{Pb}$ targets at 50 $\mathrm{MeV} /$ nucleon as a function of the scattering angle in degrees, and for a relative energy, $E_{r e l}$, between the proton and the ${ }^{7} \mathrm{Be}$ fragment equal to $0.2 \mathrm{MeV}\left(E_{x}=E_{r e l}+0.14 \mathrm{MeV}\right)$. The excitation cross sections to the p- and f-waves and to the $3^{+}$resonance are displayed. Since the $1^{+}$state has a very small width, it does not contribute appreciably for this excitation energy. Figure $3(\mathrm{~b})$ shows the same calculation, but for a relative energy of $1.2 \mathrm{MeV}$. The basic feature in these being an oscillatory pattern, characteristic of diffraction by a strong absorptive object. The measured angular distributions on the other hand are rather flat in the angular range of interest, suggesting small nuclear contribution(s).

In figure 4 we plot the angular integrated nuclear excitation cross section as a function of the relative energy and for the bombarding energy of $50 \mathrm{MeV} /$ nucleon. We see that most of the excitation cross section goes to the $1^{+}$and $3^{+}$ state; the excitation of the p- and f-waves being of much smaller magnitude. We observe that the nuclear interaction broadens the width of the $1^{+}$and $3^{+}$resonances (e.g., for the $1^{+}$resonance, $\Gamma \approx 50 \mathrm{keV}$ ).

In figure 5(a) and we show the Coulomb excitation cross sections of ${ }^{8} B$ projectiles incident on $P b$ targets at 50 $\mathrm{MeV} /$ nucleon as a function of the scattering angle in degrees, and for a relative energy, $E_{\text {rel }}$, between the proton and the ${ }^{7} B e$ fragment equal to $0.2 \mathrm{MeV}\left(E_{x}=E_{r e l}+0.14 \mathrm{MeV}\right)$. In figure $5(\mathrm{~b})$ we use $E_{r e l}=1.2 \mathrm{MeV}$. We notice that the E1 excitation dominates at low angles and that the E2 excitation becomes as strong as the E1 mode at larger angles. The peak value occurs at $\theta \sim 0.4^{\circ}$ where the $\mathrm{E} 1$ cross section is more than one order of magnitude bigger that the $\mathrm{E} 2$, and more than two orders of magnitude bigger than the $\mathrm{M} 1$ excitation. Plots for $250 \mathrm{MeV} /$ nucleon are presented in figs. 6(a) and 6(b), respectively. It is also interesting to compare these calculations with the semiclassical formula presented in ref. 14] for the E1 excitation mode, which is much easier to use. As we see in figure 7, the cross section is very well reproduced by the semiclassical formula. Diffraction effects are of minor relevance and only introduce wiggles in the cross section around the semiclassical results.

In figure 8(a) we show the Coulomb excitation cross section integrated over angles for $50 \mathrm{MeV} /$ nucleon, as a function of the relative energy of the fragments. Figure 8 (b) is for $250 \mathrm{MeV} /$ nucleon. Note that E2 excitation are dominant at large angles and hence the E2 mode contributes one fifth of the total cross section even though it is negligible at small angles (see below). And the M1 excitation is only relevant around the $1^{+}$resonance, $E_{r e l} \sim 600 \mathrm{keV}$. Comparing this figure with figure 4 we see that the nuclear contribution to the total excitation cross section is about 3 orders of magnitude smaller than the Coulomb excitation cross sections for the whole spectrum. This is a very important result, since one can neglect the nuclear excitation cross sections for practical purposes. The same applies for 250 $\mathrm{MeV} /$ nucleon.

In figs. 9(a), 9(b) and 9(c) we compare the results of our model with the angular distributions of ${ }^{8} B$ breakup on lead targets at $50 \mathrm{MeV} /$ nucleon measured by Kikuchi et al. [15] at RIKEN. We have used the acceptance (efficiency) matrix as well as angular and energy averaging procedures as discussed in Ref. 15] and provided by the RIKEN collaboration 
[15]. We plot our predictions for the contributions of the E1, E2, and of the nuclear excitation cross sections. The solid line is the sum of all three contributions, and we note the rather good agreement with the experimental data with E1 excitation solely. Our model suggest a very negligible E2 contribution as was the conclusion of Kikuchi et al. [15], where upper limits on the E2 contribution were extracted. These upper limits are indeed consistent with a preliminary analysis of the previous RIKEN data [4 that we published earlier [16].

\section{A. Conclusions}

We have constructed a simple model for ${ }^{8} B$ as well as a simple optical model for the elastic scattering of ${ }^{8} B$ plus ${ }^{208} \mathrm{~Pb}$, and used it to predict nuclear excitations as well as E1, E2 and M1 excitations of ${ }^{8} B$ at 50 and $250 \mathrm{MeV} / \mathrm{u}$. We show that nuclear excitations as well as E2 and M1 excitations are negligible for the most part of the data taken at RIKEN and at GSI.

\section{Acknowledgments}

This work was supported in part by the FAPERJ/Brazil, by the MCT/FINEP/CNPQ(PRONEX)/Brazil under contract No. 41.96.0886.00, and by the USDOE Grant No. DE-FG02-94ER40870.

[1] G. Baur, C.A. Bertulani, and H. Rebel; Nucl. Phys. A458 (1986) 188.

[2] C.A. Bertulani and G. Baur; Phys. Rep. 163 (1988) 299.

[3] Moshe Gai, Nucl. Phys. B(Sup.)38 (1995) 77.

[4] T. Motobayashi et al.; Phys. Rev. Lett. 73 (1993) 2680, and N. Iwasa et al. Jour. Phys. Soc. Jpn. 65 (1996) 1256.

[5] K. Suemerrer et al., GSI EX-171 (1996), in progress and to be published.

[6] C.A. Bertulani, Z. Phys. A356 (1996) 293.

[7] G.R. Satchler, "Direct Nuclear Reactions", Clarendon Press, Oxford 1983.

[8] C.A. Bertulani and H. Sagawa, Nucl. Phys. A588 (1995) 667.

[9] A.M. Kobos, B.A. Brown, R. Lindsay and G.R. Satchler, Nucl. Phys. A425 (1984) 205.

[10] C.A. Bertulani, P. Lotti and H. Sagawa, Phys. Rev. C (01/Jan/98 issue), in press.

[11] C.A. Bertulani and A.M. Nathan, Nucl. Phys. A554 (1993) 158.

[12] A. Winther and K. Alder, Nucl. Phys. A319 (1979) 518.

[13] G.R. Satchler, Nucl. Phys. A195 (1972) 1.

[14] C.A. Bertulani, Nucl. Phys. A587 (1995) 318.

[15] T. Kikuchi et al., Phys. Lett. B391 (1997) 261. 
[16] Moshe Gai and Carlos A. Bertulani, Phys. Rev. C52 (1995) 1706.

\section{Figure Caption}

Fig. 1 - (a) Transition densities for the bound state of ${ }^{8} B$ to the $J=1^{+}$, and $J=3^{+}$, resonances at $E_{x}=0.63$ $\mathrm{MeV}$ (dashed line), and $E_{x}=2.17 \mathrm{MeV}$ (solid line), respectively. These transition densities have been integrated over the width of the resonances. Also shown in this figure (dotted line) is the transition density obtained by the Tassie model. (b) Transition densities from the ground state of ${ }^{8} B$ to the non-resonant p- (solid curve) and f-waves (dashed curve).

Fig. 2 - (a) Real part of the transition potentials for the nuclear excitation of ${ }^{8} B$ at $50 \mathrm{MeV} / \mathrm{nucleon}$ from the ground state to the resonances at $J=1^{+}$(solid line) and $J=3^{+}$(dashed line), respectively. They are compared with the Copenhagen model (dotted line) for the transition potential. (b) Real part of the transition potentials for the nuclear excitation of ${ }^{8} \mathrm{~B}$ at $50 \mathrm{MeV} /$ nucleon from the ground state to the non-resonant p- and $\mathrm{f}$-waves.

Fig. 3 - Cross section for the nuclear excitation of ${ }^{8} \mathrm{~B}$ projectiles incident on $\mathrm{Pb}$ targets at $50 \mathrm{MeV} / \mathrm{nucleon}$ as a function of the scattering angle in degrees, and for a relative energy, $E_{\text {rel }}$, between the proton and the ${ }^{7} B e$ fragment equal to $0.2 \mathrm{MeV}$. The excitation cross sections to the $\mathrm{p}$ - (solid curve) and f-waves (dashed curves) and to the $3^{+}$ resonance (dotted curves) are displayed. (b) Same as in (a), but for $E_{\text {rel }}=1.2 \mathrm{MeV}$.

Fig. 4 - Angular integrated nuclear excitation cross section of ${ }^{8} B$ projectiles incident on lead targets at 50 $\mathrm{MeV} /$ nucleon. The cross sections for the excitation of the $1^{+}$(solid line), $3^{+}$(dashed line) resonances, and to the $p_{1 / 2}$ wave (dotted line) are shown.

Fig. 5 - (b) Coulomb excitation cross sections of ${ }^{8} \mathrm{~B}$ projectiles incident on $\mathrm{Pb}$ targets at $50 \mathrm{MeV} / \mathrm{nucleon}$ as a function of the scattering angle in degrees, and for a relative energy, $E_{\text {rel }}$, between the proton and the ${ }^{7} B e$ fragment equal to $0.2 \mathrm{MeV}$. The excitation cross sections due to M1- (dashed-dotted), E2- (dotted curve), E1- (dashed curve) excitation modes are shown. The solid curve is the sum of all these contributions. (b) Same as in (a), but for $E_{\text {rel }}=1.2 \mathrm{MeV}$.

Fig. 6 - Same as in figure 5 , but for $250 \mathrm{MeV} /$ nucleon.

Fig. 7 - Comparison between the quantal (solid curves) and the semiclassical (dashed curves) calculations of Coulomb excitation of ${ }^{8} B$ projectiles incident of lead targets for $E_{\text {rel }}=1.2 \mathrm{MeV}$ at $50 \mathrm{MeV} / \mathrm{nucleon}$ and 250 $\mathrm{MeV} /$ nucleon, respectively.

Fig. 8 - (a) Coulomb excitation cross section of ${ }^{8} B$ projectiles incident of lead targets at $50 \mathrm{MeV} / \mathrm{nucleon}$ as a function of the relative energy of the fragments, and for the M1- (dotted curve), E2- (dashed-dotted curve) and E1(dashed curve) excitation modes. The solid curve is the sum of all contributions. (b) Same as in (a), but for 250 $\mathrm{MeV} /$ nucleon.

Fig. 9 - Angular distributions of ${ }^{8} \mathrm{~B}$ breakup on lead targets at $50 \mathrm{MeV} /$ nucleon. Data are from Kikuchi et al. [15]. The separate contributions of the E1, E2, and nuclear interaction are shown. 

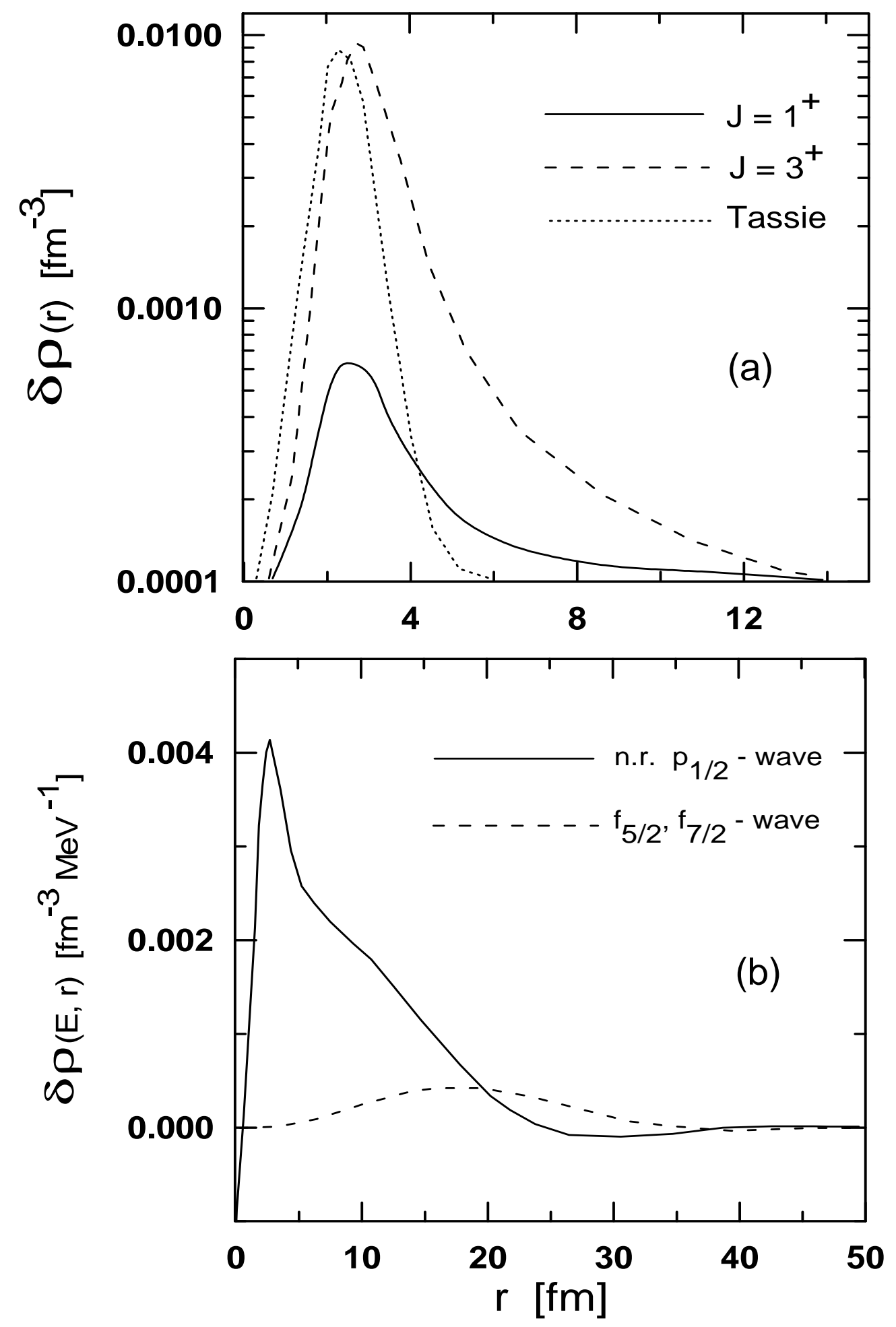

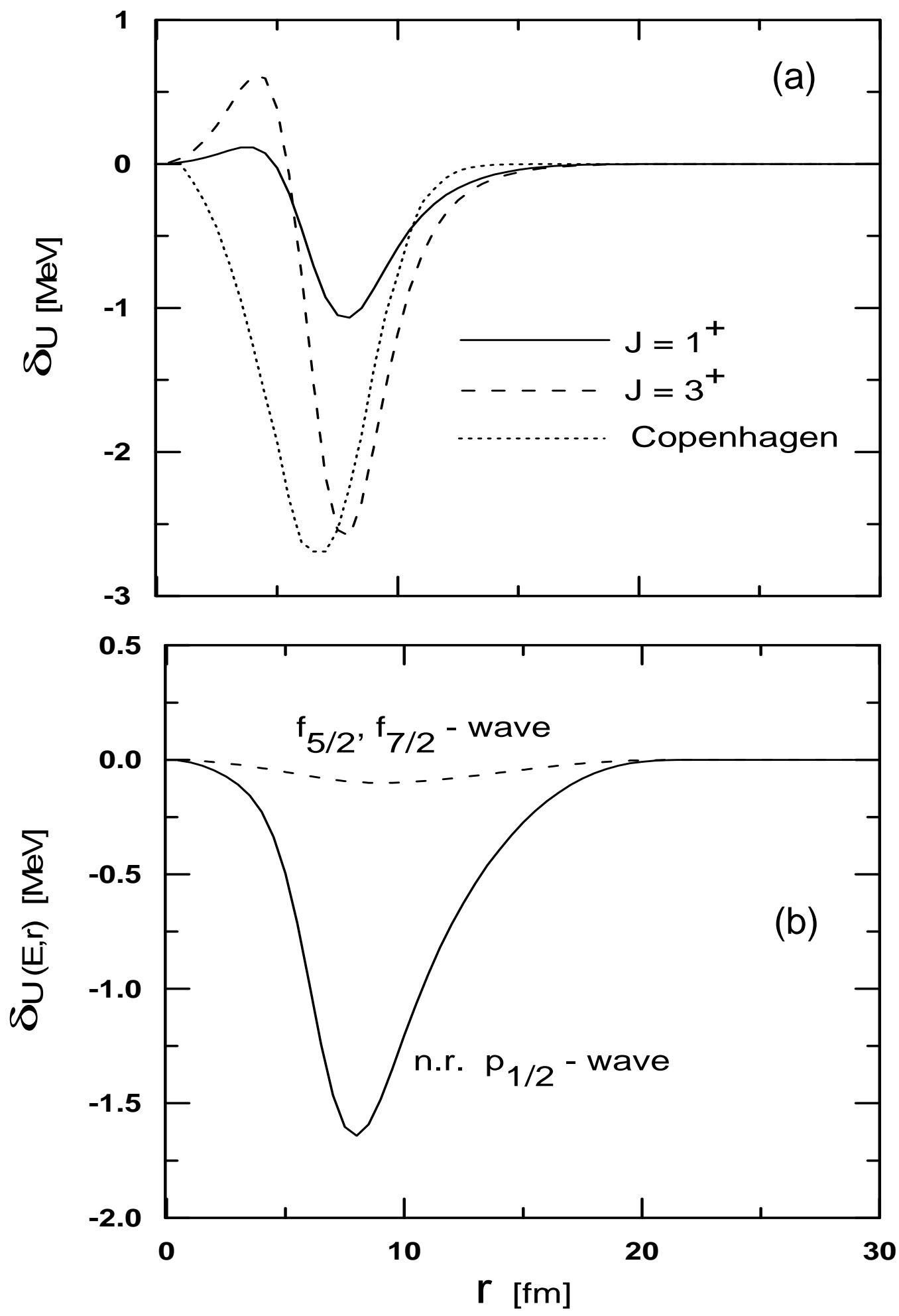


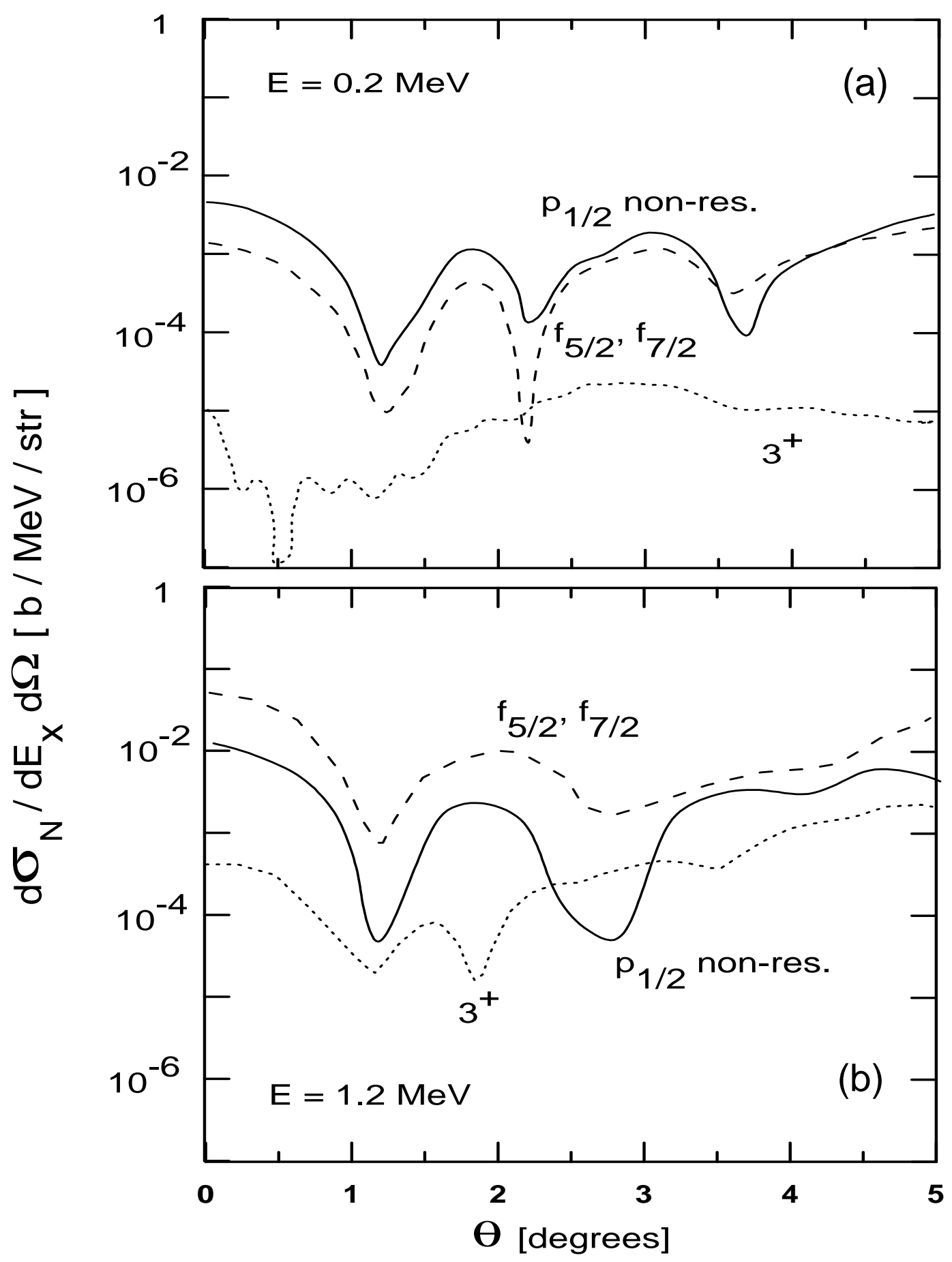




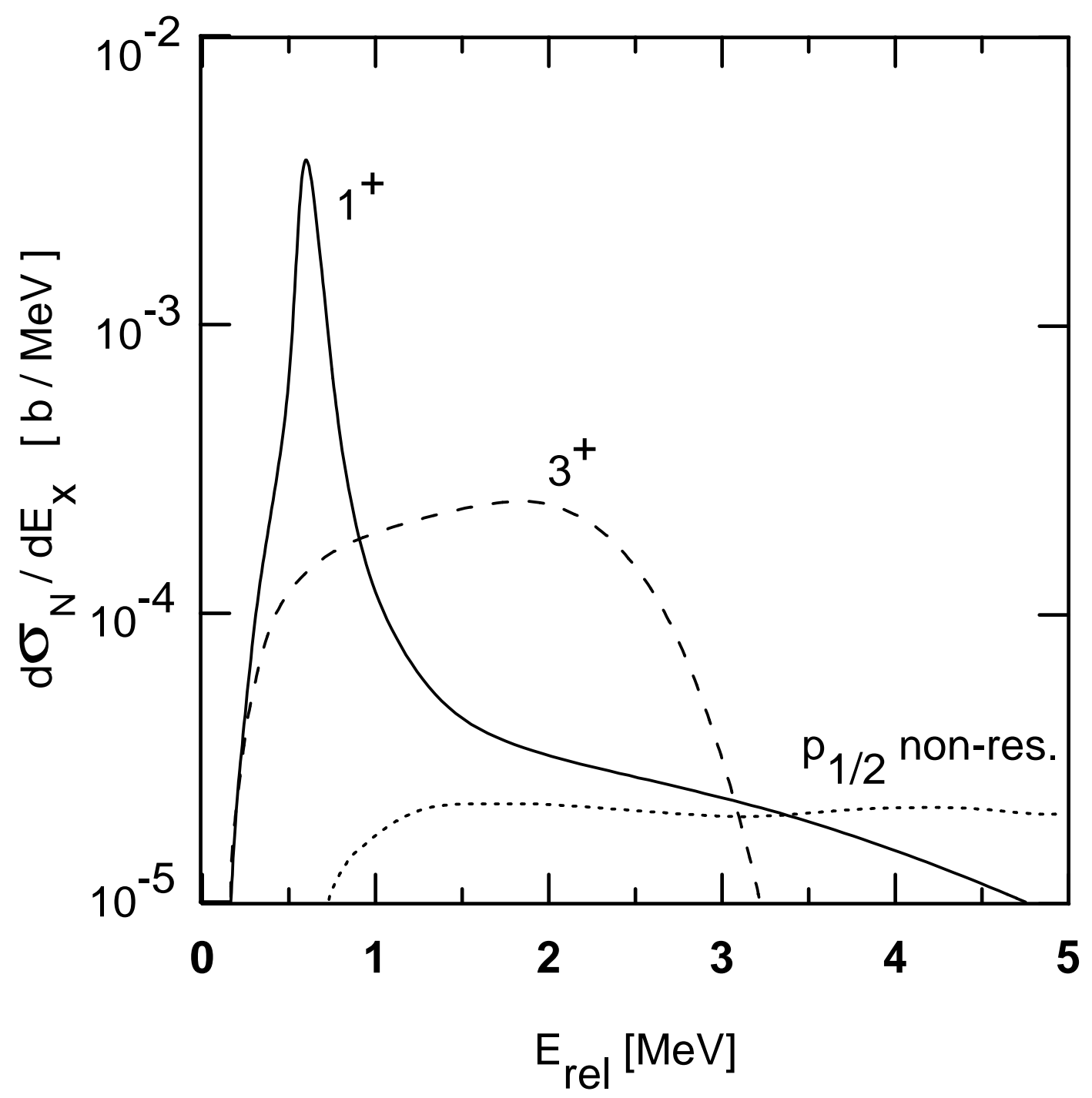




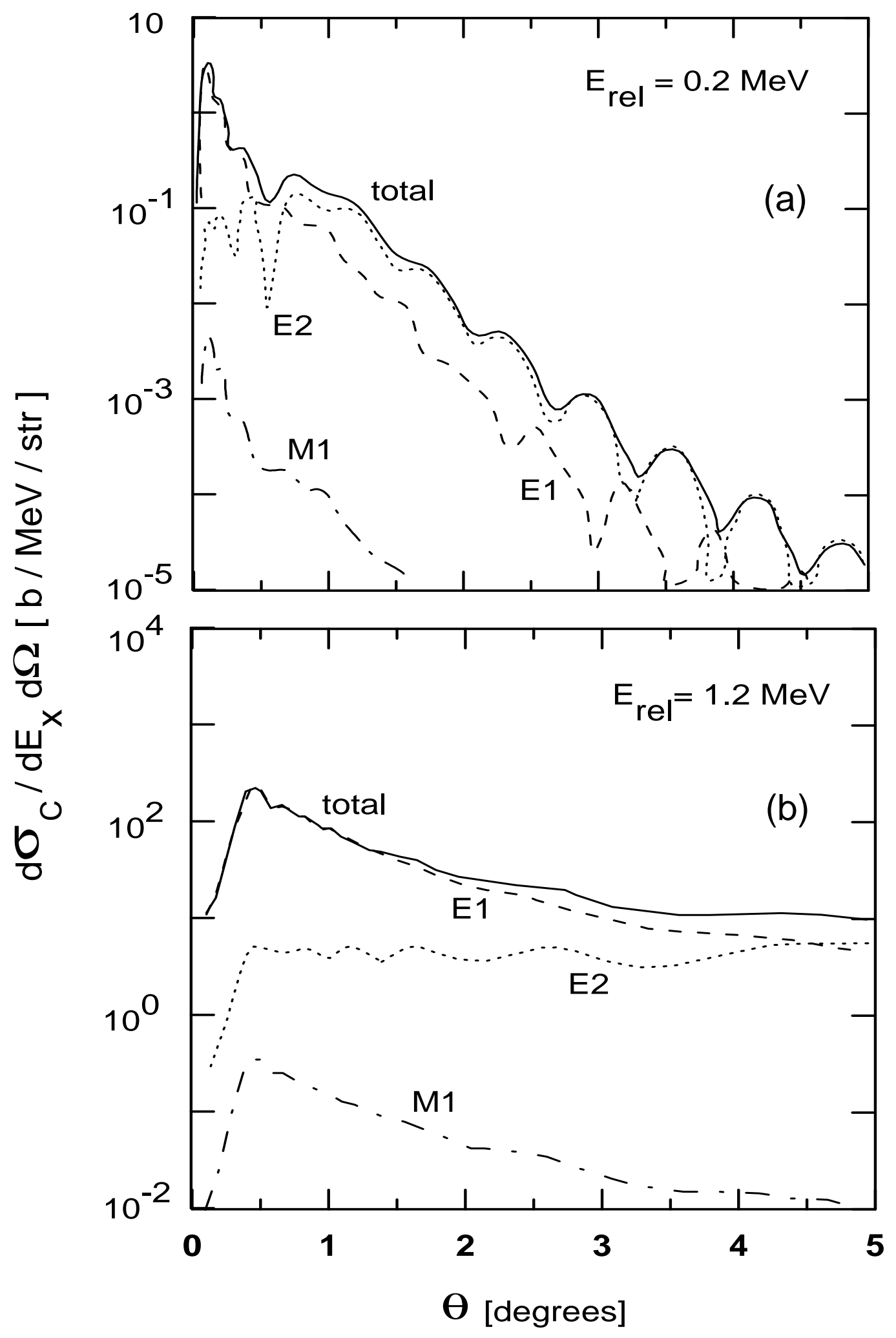




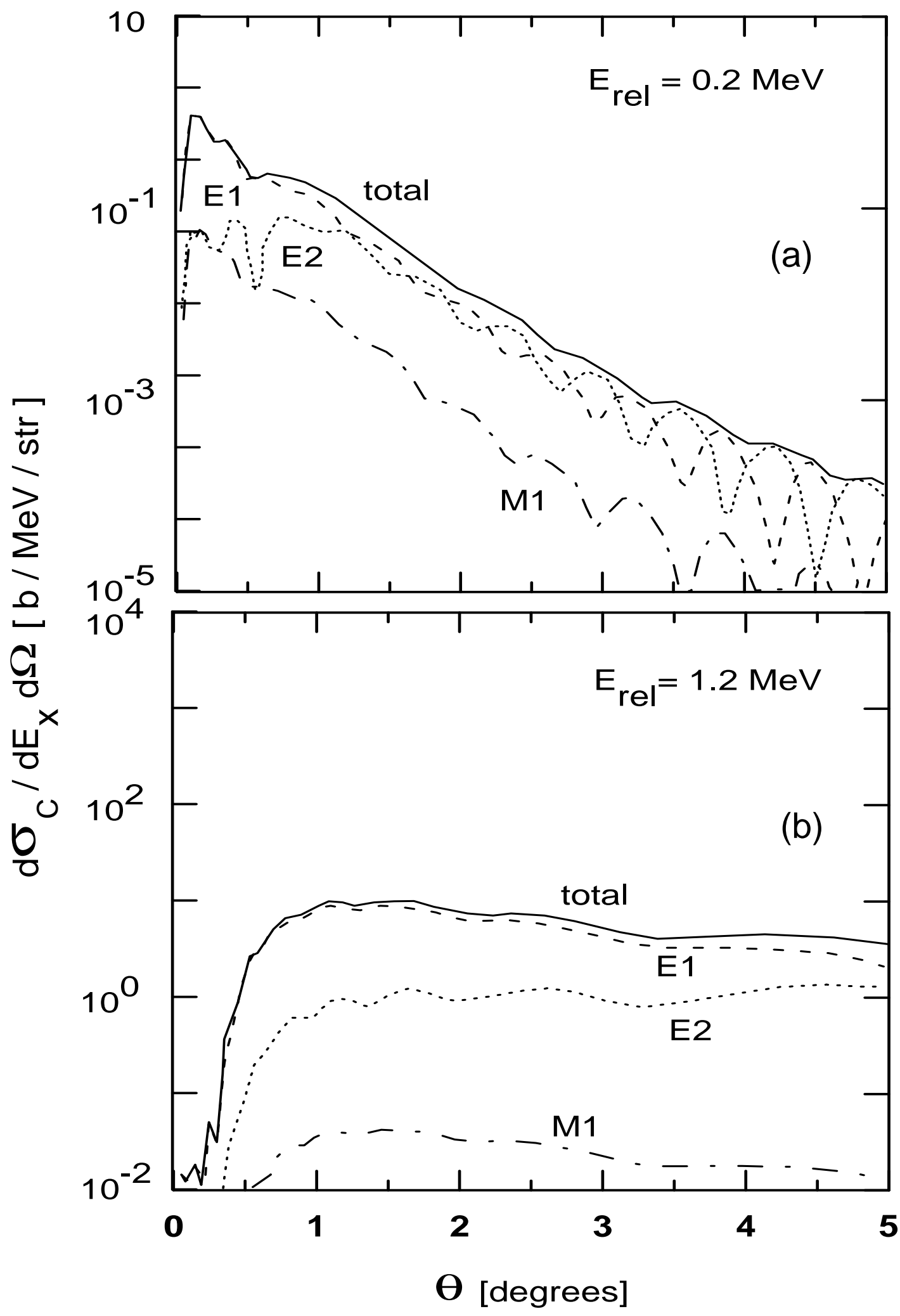




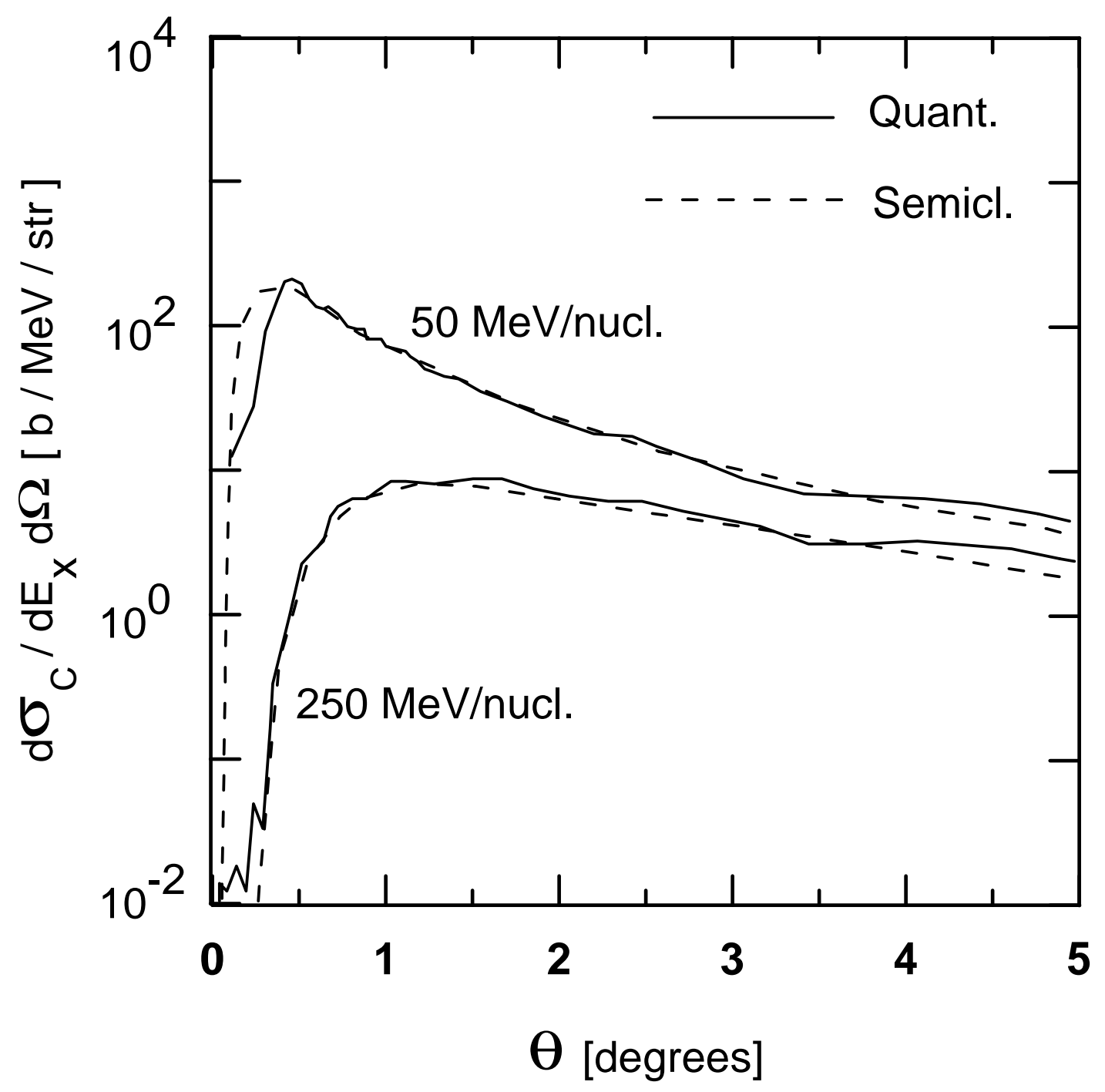




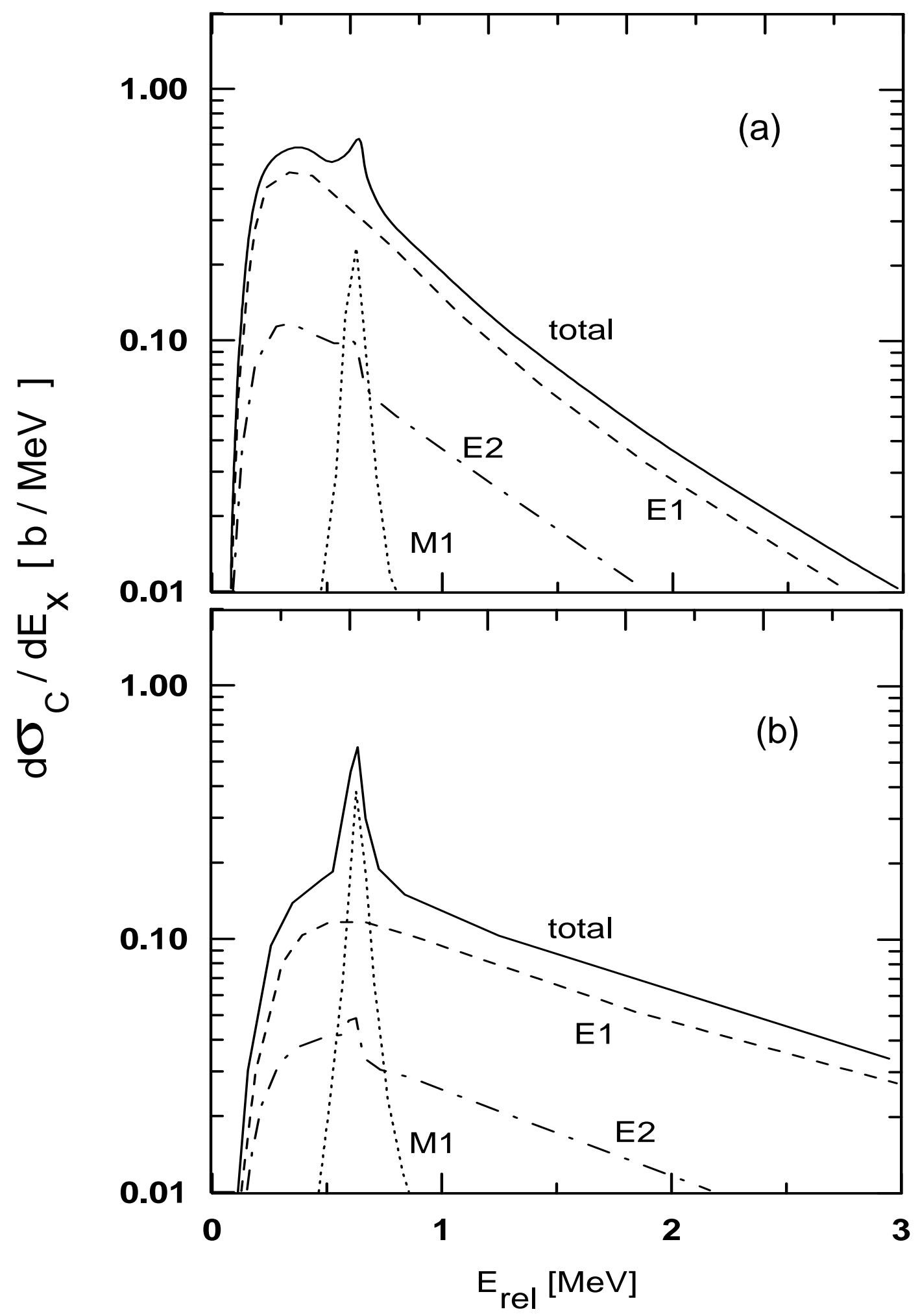




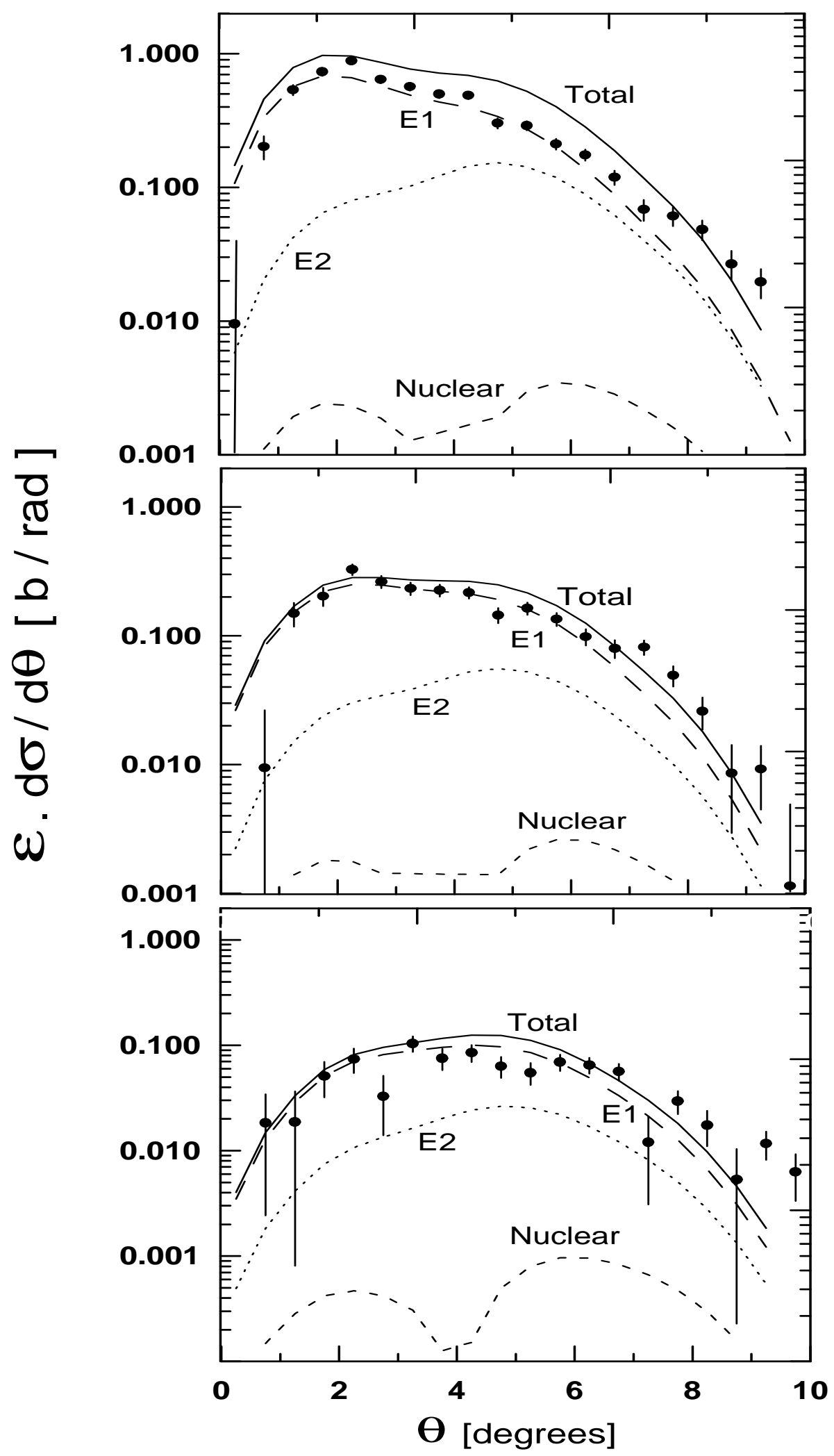

